\title{
The aid of colour on visuospatial navigation of elderly people in a virtual polyclinic environment
}

\author{
Özge Kumoğlu Süzer @ । Nilgün Olguntürk ()
}

Bilkent University, Department of Interior Architecture and Environmental Design, Faculty of Art, Design and Architecture, 06800, Bilkent, Ankara, Turkey

Correspondence

Ozge Kumoglu Suzer, Ph.D. Candidate, Bilkent

University, Department of Interior Architecture and Environmental Design, Faculty of Art, Design and Architecture, 06800, Bilkent, Ankara, Turkey.

Email: ozge.kumoglu@bilkent.edu.tr

\begin{abstract}
It is well documented that elderly people often have difficulties in finding their way in unfamiliar environments. In this study, we aimed to explore the usage of colour as visuospatial navigation tool for elderly people by testing in a virtually simulated polyclinic environment. We compared neutral, warm and cool coloured experiment settings to find out the effect of different colours on elderly people's visuospatial navigation performances. We conducted our experiment with 90 participants in two phases, each with a specific task (Route Replication and Picture Classification). The findings showed that neutral coloured setting affected participants' visuospatial navigation performances negatively compared to warm and cool coloured settings. However, there was no significant performance difference between warm and cool coloured environments. In addition to that, when they were asked to reach to the destination point, male participants spent shorter time, did fewer wrong turns, experienced fewer hesitations and traveled shorter distances compared to female participants. Currently, due to the increase in the elderly population, designing well-functioning interior spaces for the elderly has become a more pressing matter. We believe that the findings of this study will provide important clues for both interior designers and environmental psychologists.
\end{abstract}

KEYWORDS

colour, elderly, virtual reality, visuospatial navigation, wayfinding

\section{1 | INTRODUCTION}

Social, economic, scientific, technological and health care related developments have caused populations to live longer and as a result, the number of elderly people has been increasing. United Nations data from 2017 show that: "The global share of older persons (aged 60 years and over) increased from $9.2 \%$ in 1990 to $12.7 \%$ in 2017 and will continue to grow, reaching $21.3 \%$ by $2050 . "{ }^{1}$ It is up to the governments worldwide to implement legislations to meet the specialized needs of the elderly population in the fields of health care, social protection, housing and employment. ${ }^{1}$ Especially, health care is a government service used more frequently by the elderly population. Health care environments are infamous for being cold and large buildings with complex interiors and the use of a poor colour scheme.
Because of these reasons, designing interiors suited for the contemporary needs of the elderly people have become a more important issue.

Elderly people have significant difficulties in the areas of memorizing maps, navigating, route learning, map learning and place learning due to cognitive decline issues such as changes in learning abilities, short-term memory, attention and response times. ${ }^{2-12}$. Elderly people require more support than the younger-aged people for navigating by themselves, specifically in an unfamiliar environment. This can be explained by changes in the brain areas that support cognitive mapping being partially responsible for the decline in wayfinding ability that is seen in aging. ${ }^{13}$ For the elderly people with limited cognitive and physical abilities, navigational aids are important elements to improve wayfinding. 
Wayfinding is the "consistent use and organization of sensory cues from the external environment in order to reach a desired destination." 14 It is also a spatial problem solving activity comprised of three interrelated hierarchical processes: decision making (development of a plan of action), decision execution (transforming the plan into appropriate behavior at the right time and place) and information processing (comprised of environmental perception and cognition, which are the information basis of the two decision-related processes). ${ }^{15-20}$

Cognitive map is "an overall mental image or representation of the spaces and the layout of a setting" and cognitive mapping is "the mental structuring process leading to the creation of a cognitive map.". Cognitive mapping is a part of environmental perception where cognition is the source of information to make and execute a decision. ${ }^{16}$ Cognitive maps are developed to facilitate wayfinding tasks containing spatial knowledge and navigation plans, in order to be used to solve spatial problems such as wayfinding and navigation. ${ }^{14,16,21,22}$ Wayfinders generally commence by either directly perceiving the environment or working from a cognitive map, generated by the integration of the three spatial knowledge levels: landmark, procedure,and survey. ${ }^{23}$

Navigation is the coordinated and goal-directed form of movement through the environment, which involves two components; wayfinding - planning and decision-making coordinated to the distal as well as local surrounds - and locomotion/travel - body movement coordinated to the local surrounds. ${ }^{24,25}$ The first constituent of navigation is wayfinding that refers to the "cognitive coordination to the distant environment, beyond direct sensorimotor access, and includes activities such as trip planning and route choice.". The route is constructed with the perceptual knowledge of given environment by the use of visual cues as well as navigational aids. Locomotion/travel, the actual motion from the current location to the new location, changing the position of the viewpoint, can be referred as: "the perceptual-motor coordination to the local surrounds, and includes activities such as moving towards visible targets and avoiding obstacles." 26,27 The visual and structural characteristics of environments make it either easier or harder to perform various navigation tasks. ${ }^{28}$

The efficiency of wayfinding systems can be increased by using appropriate environmental design elements such as layout, landmarks and signage. The use of colour is also a valuable tool for navigation to overcome the difficulties of navigating in unfamiliar environments by helping a person develop a mental map of the indoor environment. ${ }^{29,30}$ Navigational cues can be verbal, graphic, architectural and spatial forms, and are available in both real and virtual environments (VEs). ${ }^{31}$ Previous research showed that in participant groups from all ages (including children, adults and elderly people), colour improves users' wayfinding and cognitive mapping performances..$^{29,32-36}$ It was found that colourful familiar cues improved place learning in older adults, as opposed to black and white abstract cues. ${ }^{32}$ It was also explored that the usage of focal colours improved route learning of children compared to non-focal coloured in a virtual maze. ${ }^{33}$ It is also reported that warm colours and bright accents were used to facilitate wayfinding and spatial orientation in the classrooms of 101 child care centers in Alabama. ${ }^{36}$ Colour was, therefore, concluded to have potential in enhancing the orientation of the users. In another study among university students, warm and cool coloured settings were perceived clearer, more attractive, navigable, and inviting in terms of space perception compared to neutral coloured setting. Moreover, remembrance performance turned out to be higher in the warm coloured setting. ${ }^{35}$ The effect of coloured landmarks on children's route learning ability was also studied, comparing two different sequenced colour settings and one gray setting. There was no significant difference found between the two different coloured settings, however, the route learning performance was inferior in the gray setting compared to coloured settings. ${ }^{34}$

\section{1 | Function of colour in visuospatial navigation}

Designers need to provide recognizable cues (signs, colours, etc.) for people to improve navigation within spaces. This can be seen in studies where participants were directed to search, they have shown a better understanding of an environment because they had better comprehension of the entire environment. When a person is more familiar with a setting, they are able to learn more about its overall configuration. ${ }^{37-39}$ The layout and shape of the interiors may affect wayfinding and an easier wayfinding may increase the functionality as well as satisfy qualities of a space. To ease wayfinding, designers should plan the layouts of spaces and shape interiors so that they relate to major circulation access in a consistent way. Use of colour to enhance visuospatial abilities and the emphasis of a place is very important in the built environment. Areas without colour resulted in poorer wayfinding performances and were inferior at pointing visitors to designated locations in buildings. Such non-coloured areas also caused difficulty for visitors who had to associate the environment they experienced with a two-dimensional representation. ${ }^{40}$

The interior layouts of hospitals are infamous for resembling labyrinths. Since hospitals are large buildings with complex interiors, it is quite difficult to navigate in them. ${ }^{41}$ Major aspects of wayfinding include degree of differentiation, visual access, signs and architectural legibility. ${ }^{42,43}$ These major aspects can be emphasized by the use of colour in architecture, where distinct colours can be used for landmarks aside from graphics, signage, and other visual cues to improve wayfinding. As colour was found to be a more useful aid than form, especially for special demographics such as elderly people or patients of dementia, colour-dominance is preferred over form-dominance. Differentiating colour on 
accent walls is a useful way to create a degree of differentiation in a monochromatic environment. ${ }^{44-47}$

Elderly people's abilities to distinguish unsaturated colours deteriorated gradually especially for cool hues, therefore, providing high levels of contrast for elderly people is crucial to enhance visual function. ${ }^{48,49}$ Elderly people can distinguish among highly saturated colours at the warm end of the spectrum and are less able to determine pastel blues on the cool side. The most difficult to see colours were noted as blue and purple among elderly people.

\section{2 | Virtual reality in spatial cognition research}

Virtual environments (VE), which allow the simulation of three-dimensional environments on a computer, are increasingly used methods for spatial cognition studies. ${ }^{33,50-65}$ VEs are now able to approximate closely the real world conditions due to the advancements in computer graphics. ${ }^{66}$ Although in the real world, it is difficult to control all the variables at once, and in some cases it is quite impossible, in a typical VE it is very easy to do such changes.

Virtual Reality (VR) is a collection of technologies for generating a human-computer interface that allows people to interact efficiently with, become immersed in, and to feel the presence in computerized 3D environments, while using their natural senses and motor skills in real time. ${ }^{67}$. VR is a very useful concept to researchers who are studying the spatial abilities, wayfinding and cognitive mapping. ${ }^{68-70}$ The concepts of presence, involvement, immersion and interaction are crucial to understand the physical and psychological experience of users in VR. ${ }^{71}$ Among all of those four concepts, presence is the most essential one because presence is "a subjective concept associated with psychological aspects of the user relationship to the sense of being in the VE.". ${ }^{71}$ Presence also refers to experiencing the VE without being aware of one's own actual physical existence. ${ }^{71}$ Involvement and immersion are presented as required conditions for experiencing presence. Involvement is "the psychological state experienced as result of focusing one's energy and attention on a coherent set of stimuli or meaningfully related activities and events" and immersion is "the psychological state characterized by perceiving oneself to be enveloped by, included in, and interacting with an environment that provides a continuous stream of stimuli.". ${ }^{72}$ Moreover, the immersion provided by a VE is directly related to the isolation from the real world and the quality of interaction involved within tasks and the environment in the VR system. $^{71}$

Previous research indicates that using VEs in spatial cognition-related studies is very beneficial compared to the usage of real environments, because it creates a more controllable environment which is easy to change by the controller. This flexibility is one of the main advantages of VR. ${ }^{67}$ VR is also a very valuable tool for researchers to monitor and record the behaviors that affect participants in terms of improving spatial knowledge. ${ }^{58}$ Almost any environment can be modeled and simulated in VR, which gives researchers a chance to measure the efficiency of navigation performance of the participants while they can operate in an independent way. ${ }^{55}$ Furthermore, in a well-designed VE, participants can acquire knowledge of directions and distances, ${ }^{73,74}$ develop route and survey knowledge ${ }^{75,76}$ and navigate effectively. ${ }^{77-80}$

The kind of immersion is based on the physical configuration of a VR user interface. There are three listed configurations: non-immersive (desktop-based VR), semi-immersive (such as CAVE Projection Screens), and fully-immersive (using a head-mounted display). Cave Automatic Virtual Environment (CAVE) is defined as a VE setting created through the combination of screens or projections upon a closed box to simulate an environment. In general, there are at least four screens covering the sides of the user's body in order to immerse the user into the VE. A head-mounted display (HMD) is a display worn on the head of the user to simulate a larger level of immersion for the user within the VE through the lenses that create the VE right in front of the user's eye sight. It has been stated that the physical level of immersion is dependent on how much the user can perceive the real world. It is clarified that the classification of immersion in VR increases as the perception (see, hear, touch) of the real world decreases. ${ }^{71}$

Currently, the most developed VR technology is the HMD system which is a fully immersive VR system where nothing intrudes the user's visual field except a scene in the simulated environment. A standard HMD system has two miniature displays and an optical system which projects the images from the screens to the eyes, thus presenting a stereo view of the virtual world. ${ }^{67}$ The position and orientation of the users' head is continuously measured by an integrated motion tracker. It allows the image generating computer to adjust the representation of the scene from the VE to the current view. Therefore, while the user walks and looks around the VE freely, the point of view changes constantly and the user is presented with a real-world-like experience of observing and interacting with the environment. The main advantage of the HMD system is the high sense of realism and presence that participants experience. The immersion level is also high, thus naming it as a fully immersive VR, where the user is isolated completely from the real world. ${ }^{81}$ Another advantage of the HMD system is the greater sense of depth it provides to the users with the fully immersive 3D graphics.

\section{3 | The scope of the study}

Previous studies showed that the aid of visual cues on spatial perception and wayfinding is important. However, it is still unclear how the colour scheme of an environment affects the visuospatial navigation performances of elderly people and if it is possible to create easier to navigate indoors for elderly 
people by using specific colour schemes. The aim of our study is to fill in the gap in literature regarding the effects of colour on visuospatial navigation of elderly people in a virtually simulated polyclinic environment (see Figure 1).

The null hypotheses of this study are:

i. There is no significant effect of colour on visuospatial navigation in elderly people.

ii. Visuospatial navigation performance of elderly people is independent from their gender.

\section{2 | METHOD}

\section{1 | Participants}

The randomly selected participant group consisted of 90 volunteers $(m=70.43$, std. dev. $=4.399, n=90)$, balanced as to gender; 45 females ( $m=70.49$, std. dev. $=4.324, n=45$ ) and 45 males $(m=70.36$, std. dev. $=4.422, n=45)$ to explore gender differences in the aid of colour on visuospatial navigation. The ages of the elder participant group varied between 65 and 80 . We conducted the experiment with three different participant groups of 15 females and 15 males each, for the neutral, warm and cool coloured experiment settings.

\section{2 | Instruments}

In order to eliminate those results in visuospatial navigation performance that may be determined by cognitive and functional impairments, spatial skills and depressive symptoms, several cognitive tests indicated below were conducted. Before the cognitive tests, a questionnaire asking the information on education level, handedness and visual impairment was answered by all participants. Participants selected and admitted to the next steps of the study were chosen from right handed, educated participants with at least 12 years of education and no severe visual impairment.

\subsection{1 | Ishihara's tests for colour-blindness}

The participants were tested for colour vision deficiencies by using Ishihara's Tests for Colour-Blindness. ${ }^{82}$ None of the participants were found to have colour vision deficiencies. Participants who use spectacles had the experiment by wearing the HMD on top of their glasses. Additionally, volunteers who reported having any severe visual difficulties were not admitted to the experiment.

\subsection{2 | Montreal Cognitive Assessment}

Montreal Cognitive Assesment (MoCA) is a simple but promising tool for evaluating mild cognitive impairment and early Alzheimer's disease. ${ }^{83,84}$ The MoCA assesses several cognitive domains. The short-term memory recall task (5 points) involves two learning trials of five nouns and delayed recall after approximately $5 \mathrm{~min}$. Visuospatial abilities are assessed using a clock-drawing task ( 3 points) and a three-dimensional cube copy (1 point). Multiple aspects of executive functions are assessed using an alternation task adapted from the trail-making B task (1 point), a phonemic fluency task (1 point), and a two-item verbal abstraction task (2 points). Attention, concentration and working memory are evaluated using a sustained attention task (target detection using tapping; 1 point), a serial subtraction task (3 points), and digits forward and backward (1 point each). Language is assessed using a three-item confrontation naming task with low-familiarity animals (lion, camel, rhinoceros; 3 points), repetition of two syntactically complex sentences ( 2 points), and the aforementioned fluency task. Finally, orientation to time and place is evaluated ( 6 points).

The MoCA is initially designed for North American population; therefore, the cut-off score is indicated as 26. The test was later modified specifically for Turkish population and the cut-off score is indicated as $21 .{ }^{84}$ In our experiment, the volunteers, who had less than 21 points from Turkish version of MoCA were not admitted to participate.

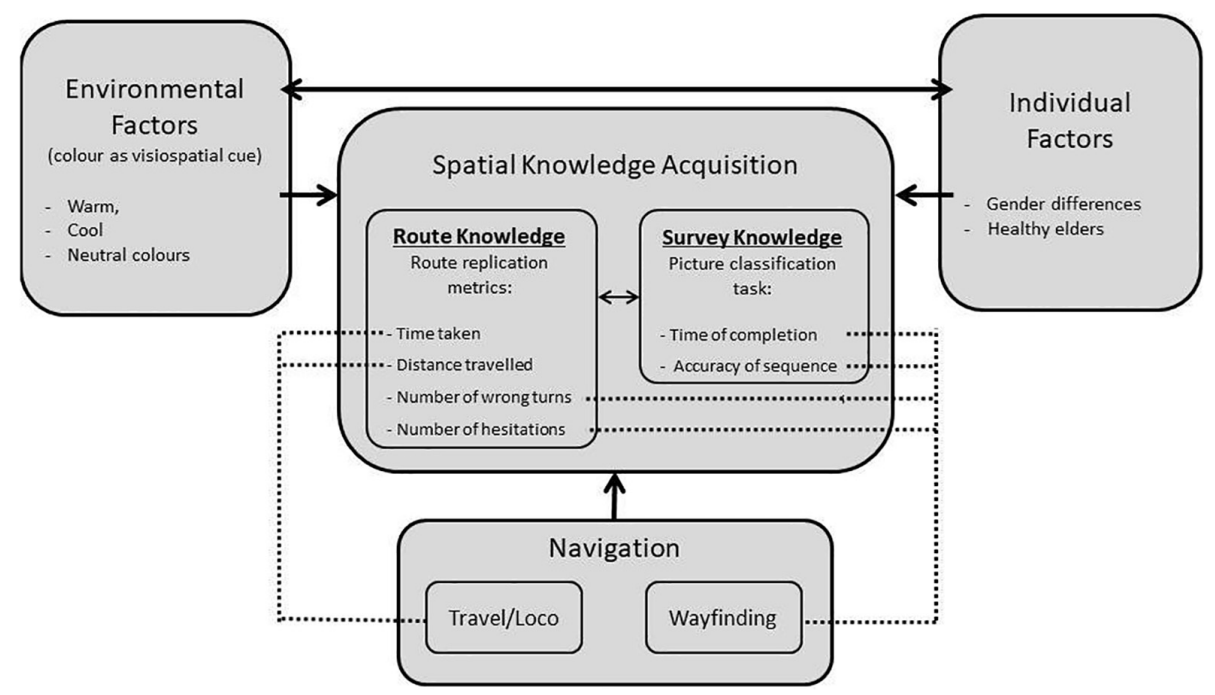




\subsection{3 | Geriatric depression scale}

It is known that depression affects physical, cognitive, functional and social impairment negatively, for this reason we conducted Geriatric Depression Scale to the participants. The Geriatric Depression Scale (GDS), is one of the most commonly used self-rated depression scales used on elderly people. ${ }^{85}$ The GDS is a 15 -item questionnaire in which participants are asked to respond by answering yes or no in reference to how they felt over the past week. It consists of the 15 items, 10 indicate the presence of depression when answered positively, while the rest (question numbers 1, 5, $7,11,13$ ) indicate depression when answered negatively.

Validity and reliability studies of GDS for Turkish population are investigated and the cut-off scores are indicated as $0-4$ are considered normal, depending on age, education and complaints; 5-8 indicate mild depression; 9-11 indicate moderate depression; and 12-15 indicate severe depression. The participants who had scores in between 0 and 4 were included to the experiment. ${ }^{86}$

\subsection{4 | Functional Activities Questionnaire}

The Functional Activities Questionnaire (FAQ) measures instrumental activities of daily living (IADLs), such as preparing meals and managing personal finances. The FAQ has 10 items concerned with performing daily tasks necessary for independent living. For each of the 10 activity, four levels ranging from dependence (scored 0) to independence (scored 3) are specified. The total score is the sum of individual item scores; higher scores reflect greater dependency. Sum scores range from 0 to 30 .

In the original version of FAQ, cut-off point of 9 (dependent in 3 or more activities) was recommended to indicate impaired function and possible cognitive impairment. ${ }^{87} \mathrm{Sim}$ ilar to MoCA, FAQ is also standardized and adopted for Turkish population and the cut-off score is indicated as 5 (dependent in 2 or more activities) for ages of 50-69 and 9 (dependent in 3 or more activities) for ages of 70 and more, to determine impaired function and possible cognitive impairment. $^{88}$ It is also indicated that the scores of FAQ increase when the age grows and education level decreases. The volunteers who cannot achieve the stated cut-off scores were not admitted to the experiment. ${ }^{88}$

\subsection{5 | Judgment of Line Orientation test}

The Judgment of Line Orientation (JLO) is a widely used test that measures a person's ability to match the angle and orientation of lines in space. Two partial line segments are presented together on one page, and the participant is asked to match the orientation of these segments to those on a multiple choice response card. The response options are made up of 11 full lines, all $18^{\circ}$ apart from one another, arranged in a semi-circle. The standardization study of JLO test is completed for Turkish population in 2004 and indicated the normative interval for 55-73 ages is in between 20 and 26 . Therefore, the participants achieved the scores in between 20 and 26 included in the experiment. ${ }^{89}$

\subsection{Experimental settings}

The hospitals in Turkey are generally either gray or beige, basically in neutral colour scheme. In addition to the mazelike structure of hospitals, this lack of colour usage makes orientation impossible for the unfamiliar visitors. For this study, to find out the positive effect of colour, the control setting is designed in neutral colour scheme (Figure 2) and to compare the effects of different colours, warm (Figure 3) and cool (Figure 4) colour settings were generated. We selected four different colours for each colour setting; the first colour was for the specific walls behind nurse stations, the second one was for the information desk, the third one was for the furniture in the waiting areas, and the fourth one was for other walls. The three-dimensional modeling of the generic hospital is created by using "Sketch Up." Once the virtual hospital environment is generated, the model is imported in to "Iris VR," which is an extension to make the VEs viewable and navigable with HTC Vive HMD.
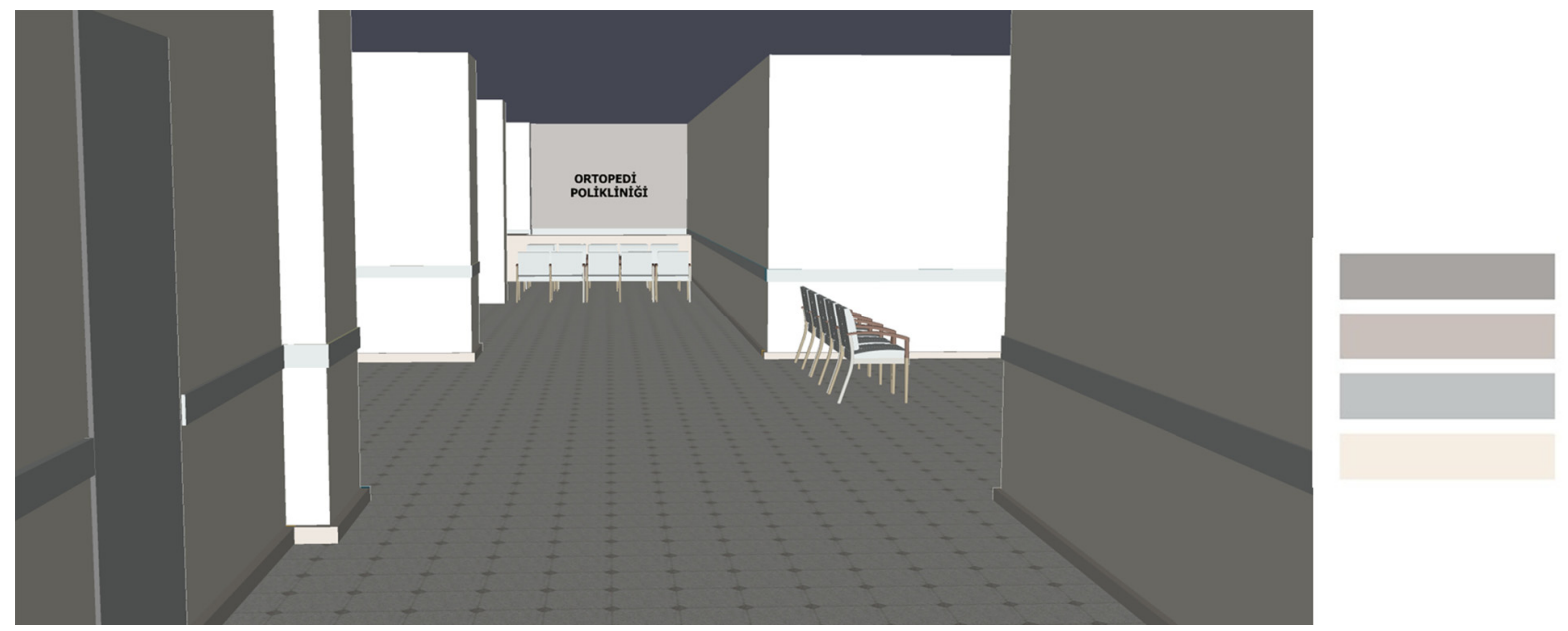

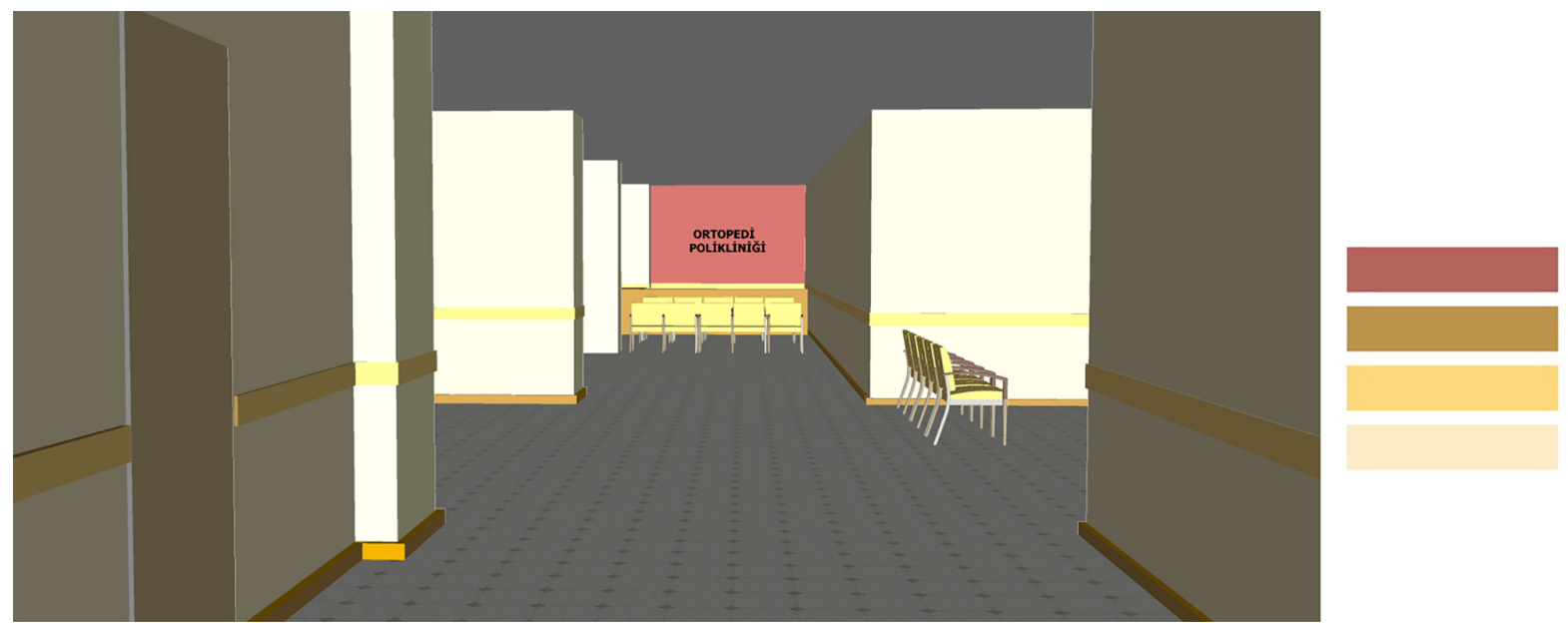

NCS S 3040-Y90R NCS S 3040-Y10R NCS S 0540-Y10R NCS S 0510-Y10R

FIGURE 3 Warm experimental settings
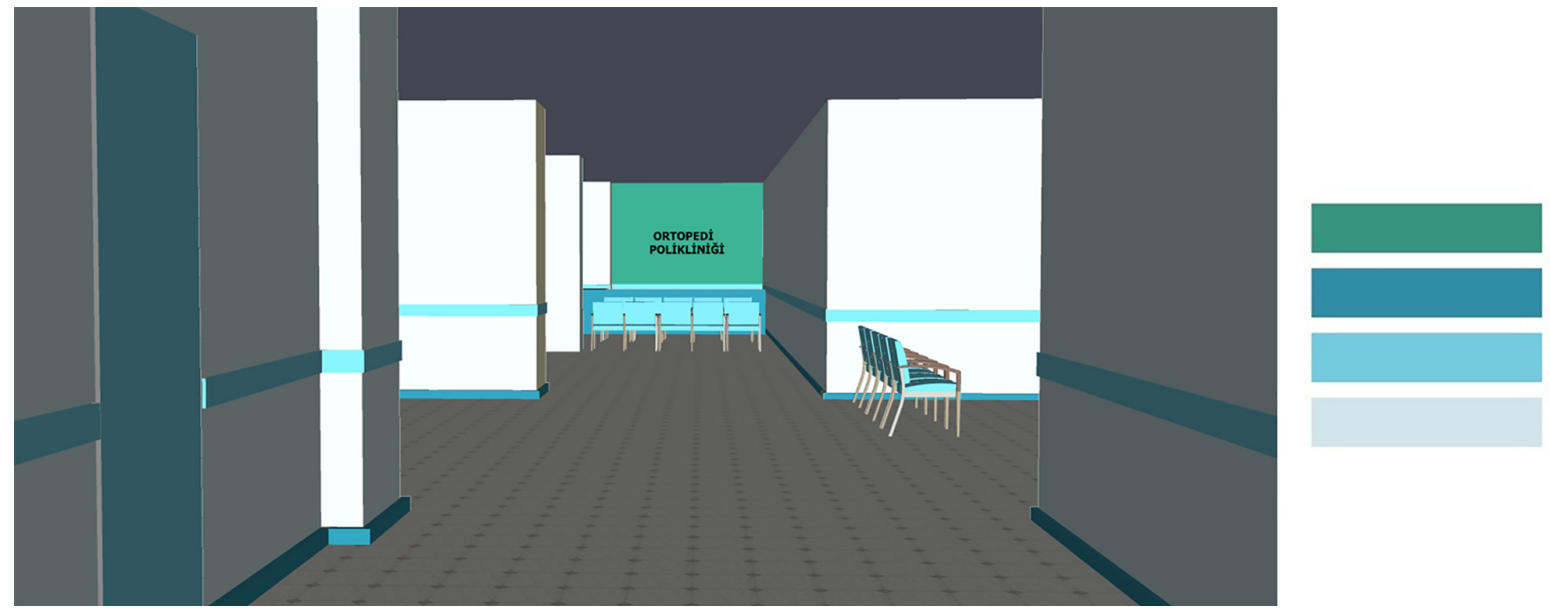

NCS S 3040-B90G

NCS S 3040-B10G

NCS S 0540-B10G

NCS S 0510-B10G

FIGURE 4 Cool experimental settings

The virtual experiment was placed on a polyclinic floor of a large-scale hospital. Providing an appropriate route representing a patient's real hospital experience was important for this study (Figure 5). As a typical route for any visitor, starting from the entrance to the selected examination room in a polyclinic floor of a hospital was simulated. Each participant was told: "Watch the route, written below, in a polyclinic prepared by computer environment. After learning the route, please wear the HMD and replicate the same route.."

\section{4 | Procedure}

The experiment consisted of three different phases.

\subsection{1 | Route replication task}

Once the participant stood in front of the computer screen and the three steps of the route replication task started:

Step 1 - Training: HTC Vive HMD was used for the study. This particular HMD has an OLED display with a resolution of $2160 \times 1200$. The refresh rate of the projected visual is $90 \mathrm{~Hz}$, and provides $110^{\circ}$ of field of view (FOV) which is very close to the human eye. Each participant wore the HTC Vive HMD and was trained to navigate in a special route prepared for training, to allow the participants to be familiarized with virtual navigation via HMD and the controller use.

Step 2 - Learning the route: Participants watched the video from a desktop computer, showing the route starting from the entrance, going through the cardiology department, ending at the examination room 3.

Step 3 - Navigating: After learning the route in Step 2, the participants wore an HTC Vive HMD and used a controller to move in the VE simulation (see Figure 6). Participants had to recall and replicate the learned route for the route replication task. Thus, every movement in the VE and the following four scores were noted during this task: (a) the time spent, (b) the number of wrong turns (when the participant navigates out of the route at a decision point, it is accepted as a "wrong turn"), (c) the number of experienced hesitations (when participants stopped and turned their heads in two different directions before starting to walk again), (d) the distance traveled during the navigation (a participant's movement through the VR environment was 


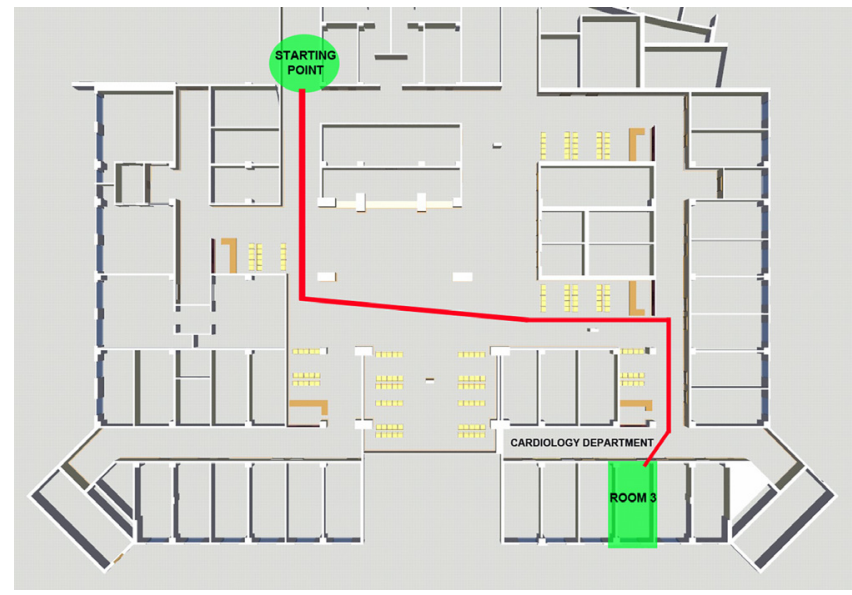

FIGURE 5 Partial plan of the polyclinic showing the route (55 meters)
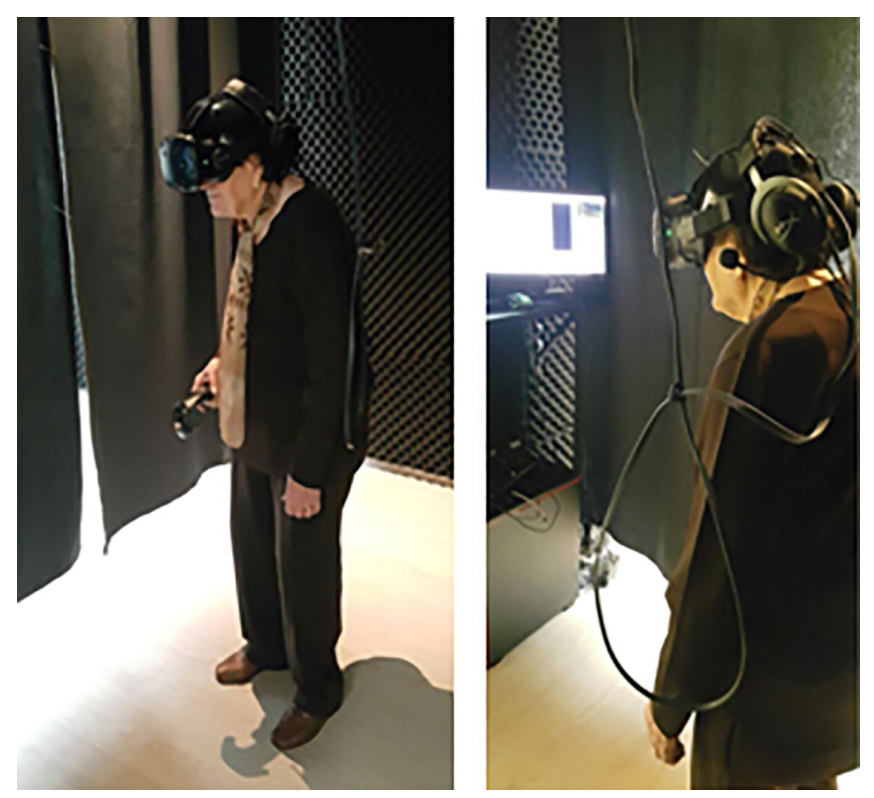

FIGURE 6 Route replication task

recorded using screen-capturing software that enabled us to replay the route taken by the participant, if needed).

\subsection{2 | Picture classification test}

This task evaluates the participants' route learning and performance of developing a cognitive map. It is known to be well performed when participants have developed clear route knowledge of the performed path. Participants were asked to sort a series of pictures taken along the route into a chronological order. The time of completion and sequence scores were measured. The score was a sequence score: 1 point was given if the photo position corresponds to the correct position in the overall sequence and half of a point is given if the position is incorrect but near a picture that immediately follows with respect to the chronological order. Zero point was for total misplacement. If all 11 pictures are ordered correctly, the maximum score was 11 .

\subsection{3 | Presence questionnaire}

Presence is defined as the subjective experience of being in one place or environment, even when the observer is physically situated in another. The assessment of sense of presence was important because the participants had to feel present in order to orient themselves in virtual reality. The effectiveness of VEs has often been linked to the sense of presence reported by users of those VEs. After navigating in the VE, the participants rated their level of presence in the VE by answering the questions in the "Presence Questionnaire."72

\section{3 | FINDINGS}

The effects of colour on visuospatial navigation were evaluated and analyzed by using the results from: Route Replication and Picture Classification tasks.

$3 \times 2$ Factorial MANOVA Test indicated that there was a statistically significant difference in visuospatial navigation performances of elderly peoples based on the colour of environmental cues $(F[12,158]=6.105, P<.0005$; Wilk's $\Lambda=0.467$, partial $\eta^{2}=0.317$ ). The Post Hoc Tests pointed out that Setting 1 (neutral coloured environment) showed a significant difference when compared to Setting 2 (warm) and Setting 3 (cool). The findings showed that neutral coloured VE affected the visuospatial navigation performances of the participants negatively compared to warm and cool coloured environments. However, there was no statistically significant difference between warm and cool coloured environments.

In addition to that, statistical difference in visuospatial navigation performance was found in terms of both route replication and picture classification tasks, based on gender $(F(6,80)=4.595, P<.0005$; Wilk's $\Lambda=0.741$, partial $\left.\eta^{2}=0.259\right)$. The findings showed that males spent shorter time, did fewer wrong turns, experienced fewer hesitations and traveled shorter distances compared to females while trying to reach their destination. Besides, males sequenced pictures more accurately and faster than females.

\section{1 | Route replication task}

\subsection{1 | The time spent}

The time spent in finding the end point for the three participant groups (the three experiment settings of Setting 1 (neutral), Setting 2 (warm) and Setting 3 (cool) were assessed by comparing the duration of the route replication task $(M=118.77, \mathrm{SD}=37.589, N=90)$. Because of the differences between three settings, exploring within females' and males' groups, $3 \times 2$ Factorial MANova was used. The main effect for colour yielded an $F$ ratio of $F(2,85)=28.9$, $P<.001$, indicating a significant difference between neutral $(M=150.33, \quad \mathrm{SD}=41.52), \quad$ warm $\quad(M=100.53, \quad \mathrm{SD}=$ 20.46) and cool coloured settings $(M=105.43, \mathrm{SD}=$ 25.18). The bar chart in Figure 7 indicates that, there is a 


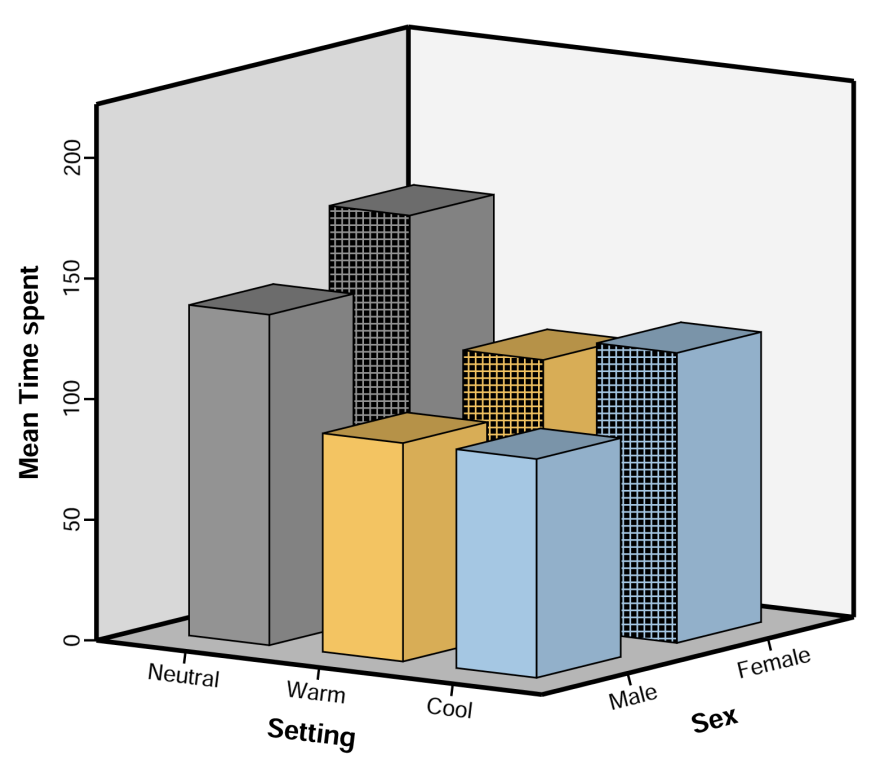

FIGURE 7 The time spent

significant difference of time spent between neutral and the other two coloured settings. However, there is not any significant difference between the warm and cool coloured settings found in terms of time spent in route replication task.

Besides, the main effect for gender on the time spent during finding the end point yielded an $F$ ratio of $\mathrm{F}(, 85)$ $=18.565, \quad P<.05$, indicating a significant difference between females $(M=131.47, \mathrm{SD}=40.347)$ and males $(M=106.07, \mathrm{SD}=30.005)$. As it is illustrated at the bar chart, females spent more time on route replication task compared to males (see Figure 7).

\subsection{2 | The number of wrong turns}

The main effect of colour on the number of wrong turns during finding the final destination yielded an $F$ ratio of $F$ $(2,85)=27.09, P<.001(M=0.33, \mathrm{SD}=0.581, N=90)$, indicating a significant difference between neutral $(M=0.83, \mathrm{SD}=0.699)$, warm $(M=0.07, \mathrm{SD}=0.254)$ and cool coloured settings $(M=0.10, \mathrm{SD}=0.305)$. As evident in Table 1, Setting 1 (neutral coloured VE) had the most number of participants taking wrong turns compared to Setting 2 (warm coloured VE) and Setting 3 (cool coloured VE). The main effect for gender on the number of wrong turns during finding the end point yielded an $F$ ratio of $F$ $(1,85)=5.344, P<.05$, indicating a significant difference between females $(M=0.44, \quad \mathrm{SD}=0.659)$ and males $(M=0.22, \mathrm{SD}=0.471)$.

First, the total number of errors in finding the final destination was assessed on the Kruskal-Wallis one-way analysis of variance test $(m=0.67$, std. dev. 0.83, $n=90)$ (Figure 6). The total number of errors means 30 participants' total number of errors for each setting in finding the final destination. The Kruskal-Wallis test indicated that the main effect of CCT on the total number of errors was not statistically significant $\left(\chi^{2}=0.08, \quad \mathrm{df}=2, \quad P=.96\right) . \quad$ The
TABLE 1 Cross-tabulation for number of wrong turns in finding the final destination in three settings

\begin{tabular}{|lllll|} 
& Neutral & Warm & Cool & Total \\
\hline Wrong turns & 20 & 2 & 3 & 25 \\
\hline No wrong turns & 10 & 28 & 27 & 65 \\
\hline Total & 30 & 30 & 30 & 90 \\
\hline
\end{tabular}

proportion of variability in the ranked dependent variable (number of errors) accounted for by the CCT variable was 0.00 . First, the total number of errors in finding the final destination was assessed on the Kruskal-Wallis one-way analysis of variance test $(m=0.67$, std. dev. $0.83, n=90)$ (Figure 6). The total number of errors means 30 participants' total number of errors for each setting in finding the final destination. The Kruskal-Wallis test indicated that the main effect of CCT on the total number of errors was not statistically significant $\left(\chi^{2}=0.08, \mathrm{df}=2, P=.96\right)$. The proportion of variability in the ranked dependent variable (number of errors) accounted for by the CCT variable was 0.00 .

\subsection{3 | The number of experienced hesitations}

The main effect for colour on the number of experienced hesitations during finding the final destination yielded an $F$ ratio of $F(2,85)=40.207, P<.001 \quad(M=0.67, \mathrm{SD}=$ $0.861, N=90$ ), indicating a significant difference between neutral $(M=1.50, \mathrm{SD}=0.900)$, warm $(M=0.20, \mathrm{SD}=$ $0.407)$ and cool coloured settings $(M=0.30, \mathrm{SD}=0.466)$. As evident in Table 2, Setting 1 (neutral coloured VE) had the most number of participants experiencing hesitations compared to Setting 2 (warm coloured VE) and Setting 3 (cool coloured VE). The main effect for gender on the number of experienced hesitations during finding the end point yielded an $F$ ratio of $F(1,85)=4.098, P<.05$, indicating a significant difference between females $(M=0.80$, $\mathrm{SD}=0.869)$ and males $(M=0.53, \mathrm{SD}=0.842)$.

\subsection{4 | The distance traveled}

The main effect for colour on the distance traveled during finding the final destination yielded an $F$ ratio of $F(2,85)$ $=18.534, \quad P<.001, \quad(M=56.34, \quad \mathrm{SD}=2.849, \quad N=90)$, indicating a significant difference between neutral $(M=58.50, \mathrm{SD}=3.93)$, warm $(M=55.10, \mathrm{SD}=0.548)$ and cool coloured settings $(M=55.43, \mathrm{SD}=1.357)$. As evident in Table 3, Setting 1 (neutral coloured VE) had the most number of participants having longer distance traveled compared to Setting 2 (warm coloured VE) and Setting 3 (cool coloured VE). The main effect for gender on the

TABLE 2 Cross-tabulation for number of experienced hesitations in finding the final destination in three settings

\begin{tabular}{lllll|} 
& Neutral & Warm & Cool & Total \\
\hline Hesitate & 26 & 6 & 9 & 41 \\
\hline Not hesitate & 4 & 24 & 21 & 49 \\
\hline Total & 30 & 30 & 30 & 90 \\
\hline
\end{tabular}


TABLE 3 Cross-tabulation for the distance traveled in finding the final destination with three settings

\begin{tabular}{|lllll|} 
& Neutral & Warm & Cool & Total \\
\hline $55 \mathrm{~m}$ & 11 & 29 & 27 & 67 \\
\hline Longer than $55 \mathrm{~m}$ & 19 & 1 & 3 & 23 \\
\hline Total & 30 & 30 & 30 & 90 \\
\hline
\end{tabular}

distance traveled during finding the end point yielded an $F$ ratio of $F(1,85)=3.957, P \leq .05$, indicating a significant difference between females $(M=56.84, \mathrm{SD}=3.464)$ and males $(M=55.84, \mathrm{SD}=1.977)$.

\section{2 | Picture classification task}

\subsection{1 | The time of completion}

The time of completion of picture classification task for the three participant groups (the three experiment settings of Setting 1 (neutral), Setting 2 (warm) and Setting 3 (cool) were assessed by comparing the duration $(M=183.84, \mathrm{SD}=$ 44.872, $N=90$ ). The main effect for colour yielded an $F$ ratio of $F(2,85)=22.796, P<.001$, indicating a significant difference between neutral $(M=218.9, \mathrm{SD}=50.329)$, warm $(M=164.23, \mathrm{SD}=27.276)$ and cool coloured settings $(M=168.4, \mathrm{SD}=31.41)$. Besides, the main effect for gender on the time of completion for picture classification task yielded an $F$ ratio of $F(1,85)=16.963, P<.05$, indicating a significant difference between females $(M=199.0$, $\mathrm{SD}=42.068)$ and males $(M=168.69, \mathrm{SD}=42.817)$ (see Figure 8).

\subsection{2 | The accuracy of sequence}

The accuracy of sequence of picture classification task for the three participant groups (the three experiment settings of Setting 1 (neutral), Setting 2 (warm) and Setting 3 (cool)

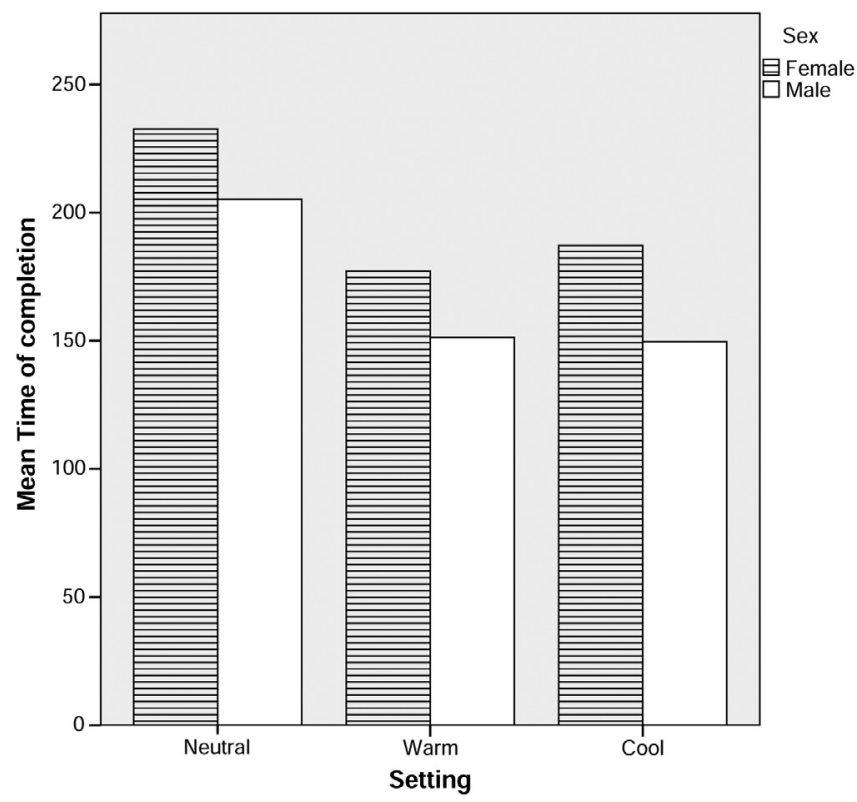

FIGURE 8 The time of completion
TABLE 4 Cross-tabulation for the sequence accuracy score in picture classification task with three settings

\begin{tabular}{|lllll|} 
& Neutral & Warm & Cool & Total \\
\hline Lower than 10 & 18 & 2 & 5 & 25 \\
\hline 10 and more than 10 & 12 & 28 & 25 & 65 \\
\hline Total & 30 & 30 & 30 & 90 \\
\hline
\end{tabular}

were assessed by comparing the accuracy scores $(M=9.98$, $\mathrm{SD}=1.081, N=90)$. The main effect for colour yielded an $F$ ratio of $F(2,85)=26.393, P<.001$, indicating a significant difference between neutral $(M=9.07, \mathrm{SD}=1.143)$, warm $(M=10.53, \mathrm{SD}=0.629)$ and cool coloured settings $(M=10.33, \mathrm{SD}=0.758)$. As evident in Table 4, Setting 1 (neutral coloured VE) had the most number of participants having lower scores compared to the participants who had Setting 2 (warm coloured VE) or Setting 3 (cool coloured $V E)$. The main effect for gender on the accuracy of sequence score for picture classification task yielded an $F$ ratio of $F$ $(1,85)=6.181, P<.05$, indicating a significant difference between females $(M=9.76, \quad \mathrm{SD}=1.069)$ and males $(M=10.20, \mathrm{SD}=1.057)$.

\section{4 | DISCUSSION}

Various aspects of the possible aid of colour to elderly peoples' visuospatial navigation performance in a virtually simulated polyclinic environment, including effects of different colour schemes in terms of route learning and cognitive mapping process were explored via using HTC Vive HMD. It was hypothesized that there are different impacts of alternating preset colour settings on visuospatial navigation performances of elderly people. Three different colour schemes (neutral, warm and cool) were compared considering two different tasks: route replication task and picture classification task. Route replication task was evaluated under four metrics; the time spent finding the end point, the number of wrong turns, the number of experienced hesitations and the distance traveled. Additionally, picture classification task was measured by two metrics: the time of completion and the accuracy of sequence.

The current research found that coloured visuospatial cues ease navigation of elderly people compared to neutral by decreasing the time spent, reducing the number of wrong turns, minimizing hesitations experienced, reducing the distance traveled. However, there was no significant difference found between warm and cool coloured visuospatial cues in terms of spatial navigation performance of elderly people. There is no research found in the literature with the focus on the effects of colour on wayfinding in cognitively healthy elderly participant groups, however, there is literature indicating that colour may be expected to improve route learning and cognitive mapping performances in wayfinding research concentrating on different age demographics, mostly adults 
and children, and elderly with dementia or Alzheimer's disease and results of the statistical tests confirmed this, showing various specific effects. ${ }^{29,30,33-36,40,46,90-93}$

The effects of coloured landmarks on wayfinding performances of children were explored in a real school environment, by comparing gray and coloured landmark usage. ${ }^{34}$ They found no significant difference between the settings of coloured landmarks, however, the wayfinding performance was found to be inferior in the setting with gray landmarks compared to the coloured sets. In the current study, even though the participant group's age demographics are different, the results were found to be very similar. The cool colour scheme including the tones of blue and the warm colour scheme including the tones of red were found to be improving the navigation performance of elderly people, while the neutral setting consisting of tones of gray and beige were found to cause inferior performances. The use of colour and lighting in hospital environments were also studied and the conclusion was that the gray and beige colours minimized the attention and concentration of people, which may have translated into an inferior navigation performance in the current study. ${ }^{29}$ Additionally, previous literature noted that the response of people is different toward colours based on their culture, ${ }^{94-96}$ and it should be considered that the current study was conducted in the Turkish culture with Turkish participants.

Additionally, the gender difference was also found on the visuospatial navigation within different coloured VEs. The results showed that females; spent more time, had more wrong turns, experienced more hesitations, and traveled longer distances compared to males. There is no consensus in literature on gender difference affecting spatial abilities. The results of this study are parallel to the results obtained in previous research $^{52,54,62,65,97-105}$ regarding the gender difference favoring males, affecting wayfinding and spatial navigation performances. However, certain studies in literature have found no gender difference or favored females in spatial performances. ${ }^{34,35,106-108}$

No significant gender difference was discovered in wayfinding performance of primary school children. ${ }^{34}$ In this current study, we found gender differences to be significant for the elderly participants between the ages 65 and 80 , favoring males. The gender differences may be surfacing a result of the experience of living in a specific culture for a long amount of time. Therefore, it is difficult to come to a clear cut conclusion on the effects of gender on visuospatial navigation due to the limited number of studies in this field regarding elderly people. The perception of gender roles also differ in between cultures and this fact has to be taken into consideration when reviewing this study. ${ }^{109,110}$

\section{I CONCLUSION AND SUGGESTIONS FOR FUTURE RESEARCH}

The major aim of this study was to fill in the gap in literature regarding the effects of colour on visuospatial navigation of elderly people in a virtually simulated polyclinic environment and, therefore, the results of current research provide data for designing more functional and recognizable spaces for the elderly people. The use of colour in the built environment to improve visuospatial abilities and navigation is crucial for special demographics such as elderly people or patients of dementia. Increasing elders' population should not be ignored and researchers, environmental psychologists, gerontologists, interior wayfinding designers, architects should collaborate to provide safe, functional and more navigable environments for the elderly people. Further spatial cognition research can be conducted in a VE through the use of a HMD, similar to the one in this study to reduce the cost of experiments both financially and physically, providing a safe, realistic feasible and easy to access alternative experience to real environments.

\section{ACKNOWLEDGMENTS}

This research was conducted as a part of $\mathrm{PhD}$ thesis of the first author in Bilkent Üniversitesi.

\section{ORCID}

Özge Kuтоğlu Süzer (10 https://orcid.org/0000-0002-0108-1020

Nilgün Olguntürk (1D) https://orcid.org/0000-0001-7947-1101

\section{REFERENCES}

[1] United Nations. World Population Ageing: Economic \& Social Affairs http://www.un.org/en/development/desa/population/publications/pdf/ ageing/WPA2017_Highlights.pdf. Updated December 10, 2017. Accessed March 22, 2018.

[2] Allain P, Nicoleau S, Pinon K, et al. Executive functioning in normal aging: a study of action planning using the zoo map test. Brain Cogn. 2005;57:4-7.

[3] Barrash J. Age related decline in route learning ability. Dev Neuropsychol. 1994;10(3):189-201.

[4] Iaria G, Palermo L, Committeri G, Barton J. Age differences in the formation and use of cognitive maps. Behav Brain Res. 2009;196:187-191.

[5] Jansen P, Schmelter A, Heil M. Spatial knowledge acquisition in younger and elderly adults. Exp Psychol. 2010;57:54-60.

[6] Lipman P, Caplan L. Adult age differences in memory for routes: effects of instruction and spatial diagram. Psychol Aging. 1992;7:435-442.

[7] Mahmood O, Adamo D, Briceno E, Moffat S. Age differences in visual path integration. Behav Brain Res. 2009;205:88-95.

[8] Moffat S, Resnick S. Effects of age on virtual environment place navigation and allocentric cognitive mapping. Behav Neurosci. 2002;116(5): 851-859.

[9] Rodgers M, Sindone J, Moffat S. Effects of age on navigation strategy. Neurobiol Aging. 2012;33:202.e15-202.e 22.

[10] Salthouse T, Siedlecki K. Efficiency of route selection as a function of adult age. Brain Cogn. 2007;63:279-286.

[11] Sjölinder M, Höök K, Nilsson L, Andersson G. Age differences and the acquisition of spatial knowledge in a three-dimensional environment: evaluating the use of an overview map as a navigation aid. Int $J$ Human-Computer Stud. 2005;63:537-564.

[12] Wilkniss S, Jones M, Korol D, Gold P, Manning C. Age-related differences in an ecologically based study of route learning. Psychol Aging. 1997;12:372-375.

[13] Moffat S, Elkins W, Resnick S. Age differences in the neural systems supporting human allocentric spatial navigation. Neurobiol Aging. 2006;27: 965-972. 
[14] Lynch K. The Image of the City. Cambridge, MA: MIT Press; 1960.

[15] Abu-Obeid N. Abstract and scenographic imagery: the effect of environmental form on wayfinding. J Environ Psychol. 1998;18:159-173.

[16] Arthur P, Passini R. Wayfinding. New York, NY: McGraw-Hill Book Co; 1992.

[17] Golledge R. Wayfinding Behavior. Baltimore, MD: Johns Hopkins University Press; 1999.

[18] Lovas G. Models of wayfinding in emergency evacuations. Eur J Oper Res. 1998;105:371-389.

[19] Passini R. Wayfinding in Architecture. New York, NY: Van Nostrand Reinhold; 1984.

[20] Rovine M, Weisman G. Sketch-map variables as predictors of way-finding performance. J Environ Psychol. 1989;9(3):217-232.

[21] Garling T, Book A, Lindberg E. Cognitive mapping of large-scale environments: the interrelationship of action plans, acquisition, and orientation. Environ Behav. 1984;16:3-34.

[22] Kitchin R. Cognitive maps: what are they and why study them? J Environ Psychol. 1994;14:1-19.

[23] Chen J, Stanney K. A theoretical model of wayfinding in virtual environments: proposed strategies for navigational aiding. Presence Teleop Virt. 1999;8:671-685.

[24] Montello D. Navigation. In: Shah P, Miyake A, eds. The Cambridge Handbook of Visuospatial Thinking. Cambridge, UK: Cambridge University Press; 2005;257-294

[25] Montello D, Sas C. Human factors of wayfinding in navigation. In: Karwowski W, ed. International Encyclopaedia of Ergonomics and Human Factors. Boca Raton, FL: CRC/Taylor \& Francis; 2006;1-17.

[26] Montello D, Freundschuh S. Cognition of geographic information. In: McMaster R, Usery E, eds. A Research Agenda for Geographic Information Science. Boca Raton, FL: CRC Press; 2005;61-91.

[27] Zhang X. A multiscale progressive model on virtual navigation. Int $J$ Human-Computer Stud. 2008;66:243-256.

[28] Karimi H. Indoor wayfinding and navigation. Boca Raton, FL: Taylor and Francis (CRC); 2015.

[29] Dalke H, Little J, Niemann E, et al. Colour and lighting in hospital design. Optics and Laser Technology 2006;38(4-6):343-365.

[30] Holscher C, Meilinger T, Vrachliotis G, Brosamle M, Knauff M. Up the down staircase: wayfinding strategies in multi-level buildings. $J$ Environ Psychol. 2006;26:284-299.

[31] Sun C, de Vries B. Automated human choice extraction for evacuation route prediction. Automation Constr. 2009;18:751-761.

[32] Davis R, Therrien B. Cue color and familiarity in place learning for older adults. Res Gerontol Nurs. 2012;5:138-148.

[33] Farran E, Courbois Y, Van Herwegen J, Cruickshank A, Blades M. Colour as an environmental cue when learning a route in a virtual environment: typical and atypical development. Res Dev Disabil. 2012;33:900-908.

[34] Helvacıoğlu E, Olguntürk N. Colour contribution to children's wayfinding in school environments. Optics Laser Technol. 2011;43:410-419.

[35] Hidayetoglu M, Yildirim K, Akalin A. The effects of color and light on indoor wayfinding and the evaluation of the perceived environment. $J$ Environ Psychol. 2012;32:50-58.

[36] Read M. Use of color in child care environments: application of color for wayfinding and space definition in Alabama child care environments. Early Childhood Educ J. 2003;30:233-239.

[37] Dogu U, Erkip F. Spatial factors affecting wayfinding and orientation: a case study in a shopping mall. Environ Behav. 2000;32:731-755.

[38] Haq S, Zimring C. Just down the road a piece: the development of topological knowledge of building layouts. Environ Behav. 2003;35:132-160.

[39] Park Y, Guerin D. Meaning and preference of interior color palettes among four cultures. J Interior Design. 2002;28:27-39.

[40] Werner W, Schindler W. The role of spatial reference frames in architecture. Environ Behav. 2004;36:461-482.

[41] Tofle R, Schwarz B, Yoon S, Max-Royale A, Des M. Color in healthcare environments - a research report. United States of America: The Coalition for Health Environments Research, San Francisco, CA; 2004.

[42] Garling T. Environmental Orientation during Locomotion. Swedish Council of Building Research: Stockholm, Sweden; 1984.

[43] Weisman D. Evaluating architectural legibility: wayfinding in the built environment. Environ Behav. 1981;13:189-204.

[44] Evans G, Fellows J, Zorn M, Doty K. Cognitive mapping and architecture. J Appl Psychol. 1980;65:474-478.
[45] Goldstein L, Oakley D. Colour versus orientation discrimination in severely brain-damaged and normal adults. Cortex. 1986;22:261-266.

[46] Kaplan R. The green experience. In: Kaplan S \& Kaplan R, eds. Humanscape: Environments for People. Ann Arbor, MI: Ulrich's Books; 1982: 186-193.

[47] Malkin J. Hospital Interior Architecture: Creating Healing Environments for Special Patient Populations. New York, NY: Van Nostrand Reinhold Co., Inc.; 1992.

[48] Brawley E. Designing for Alzheimer's Disease: Strategies for Creating Better Care Environments. New York, NY: Wiley; 1997.

[49] Cooper B, Ward M, Gowland C, McIntosh J. The use of the lanthony new color test in determining the effects of aging on color vision. $J$ Gerontol. 1991;46:320-324.

[50] Blackman T, Mitchell L, Burton E, et al. The accessibility of public spaces for people with dementia: a new priority for the 'open city'. Disabil Soc. 2003;18:357-371.

[51] Cohen R, Schuepfer T. The representation of landmarks and routes. Child Dev. 1980;51:1065-1071.

[52] Cubukcu E, Nasar J. Influence of physical characteristics of routes on distance cognition in virtual environments. Environ Plan B Plan Design. 2005;32:777-785

[53] Cubukcu E, Nasar J. Relation of physical form to spatial knowledge in large scale virtual environments. Environ Behav. 2005;37:397-417.

[54] Devlin A, Bernstein J. Interactive wayfinding: use of cues by men and women. J Environ Psychol. 1995;15:23-38.

[55] Jansen-Osmann P, Wiedenbauer G. Wayfinding performance in and the spatial knowledge of a color-coded building for adults and children. Spatial Cogn Comput. 2004;4:337-358.

[56] Moffat S, Hampson E, Hatzipantelis M. Navigation in a virtual maze: gender differences and correlation with psychometric measures of spatial ability in humans. Evol Human Behav. 1998;19(2):73-87.

[57] Moffat S, Zonderman A, Resnick S. Age differences in spatial memory in a virtual environment navigation task. Neurobiol Aging. 2001;22:787-796.

[58] Morganti F, Carassa A, Geminiani G. Planning optimal paths: a simple assessment of survey spatial knowledge in virtual environments. Comp Human Behav. 2007;23:1982-1996.

[59] Omer I, Goldblatt R. The implications of inter-visibility between landmarks on wayfinding performance: an investigation using a virtual urban environment. Comp Environ Urban Syst. 2007;31:520-534.

[60] Ruddle R, Payne S, Jones D. Navigating large-scale "desk-top" virtua buildings: effects of orientation aids and familiarity. Presence Teleop Virt. 1998;7:179-192.

[61] Ruddle R, Péruch P. Effects of proprioceptive feedback and environmental characteristics on spatial learning in virtual environments. Int $J$ Human-Comp Stud. 2004;60:299-326.

[62] Sandstrom N, Kaufman J, Huettel S. Males and females use different distal cues in a virtual environment navigation task. Cogn Brain Res. 1998;6: 351-360.

[63] Tang C, Wu W, Lin C. Using virtual reality to determine how emergency signs facilitate wayfinding. Appl Ergon. 2009;40:722-730.

[64] Umemura H, Watanabe H, Matsuoka K. Investigation of the model for path selection behavior in open space. Electronics Commun Japan (Part III: Fundam Electronic Sci). 2005;88:18-26.

[65] Tlauka M, Brolese A, Pomeroy D, Hobbs W. Gender differences in spatial knowledge acquired through simulated exploration of a virtual shopping centre. J Environ Psychol. 2005;25:111-118.

[66] Hardiess G, Mallot H, Meilinger T. Virtual reality and spatial cognition. Int Encyclopedia Social Behav Sci. 2015;25:133-137.

[67] Vilar E, Rebelo F. Virtual reality in wayfinding studies. Proceedings of 2nd International Conference on Applied Human Factors and Ergonomics jointly with 12th International Conference on Human Aspects of Advanced Manufacturing. 2008; Las Vegas, NV.

[68] Richardson A, Montello D, Hegarty M. Spatial knowledge acquisition from maps and from navigation in real and virtual environments. Memory Cogn. 1999;27:741-750.

[69] Sharlin E, Watson B, Sutphen S, Liu L, Lederer R, Frazer J. A tangible user interface for assessing cognitive mapping ability. Int $J$ Human-Computer Stud. 2009;67:269-278.

[70] Wu A, Zhang W, Zhang X. Evaluation of wayfinding aids in virtual environment. Int J Human-Computer Interact. 2009;25:1-21. 
[71] Rebelo F, Duarte E, Noriega P, Soares M. 24 Virtual reality in consumer. Human Factors Ergonomics Consumer Product Design Meth Techn. 2011;964-982.

[72] Witmer B, Singer M. Measuring presence in virtual environments: a presence questionnaire. Presence Teleop Virt. 1998;7:225-240.

[73] Albert W, Rensink R, Beusmans J. Learning relative directions between landmarks in a desktop virtual environment. Spatial Cogn Comput. 1999; 1:131-144.

[74] Willemsen P, Gooch A. Perceived egocentric distances in real image based and traditional virtual environments. In: Macedonia M, Susumu T, eds. Proceedings of the IEEE virtual reality 2002. Orlando, FL. Computer Society; 2002:275-276.

[75] Gillner S, Mallot H. Navigation and acquisition of spatial knowledge in a virtual maze. J Cogn Neurosci. 1998;10:445-463.

[76] Jansen-Osmann P. Using desktop virtual environments to investigate the role of landmarks. Comp Human Behav. 2002;18:427-436.

[77] Darken R, Sibert J. Navigating large virtual spaces. Int $J$ Human-Computer Interact. 1996;8:49-71.

[78] Ruddle R, Payne S, Jones D. The effects of maps on navigation and search strategies in very-large-scale virtual environments. J Exp PsycholAppl. 1999;5:54-75.

[79] Sancaktar I, Demirkan H. Spatial updating of objects after rotational and translational body movements in virtual environments. Comp Human Behav. 2008;24:2682-2696.

[80] Sancaktar İ. Exploring Vertical Navigation within a Virtual Environment: A Staircase Experience [unpublished doctoral thesis]. Ankara, Turkey: Bilkent University; 2012.

[81] Gutierrez M, Vexo F, Thalmann D. Stepping into Virtual Reality. Santa Clara, CA: Springer Science \& Business Media; 2008.

[82] Ishihara S. Tests for Color-Blindness. Tokyo, Japan: Kanehara; 1960.

[83] Nasreddine Z, Philips N, Bedirian V, et al. The Montreal cognitive assessment, MoCA: a brief screening tool for mild cognitive impairment. J Am Geriatr Soc. 2005;53:695-699.

[84] Selekler K, Cangöz B, Uluç S. Montreal Bilişsel Değerlendirme Ölçeği'nin (MOBİD) Hafif Bilişsel Bozukluk ve Alzheimer Hastalarını ayırdedebilme gücünün incelenmesi. Türk Geriatri Dergisi. 2010;13: 166-171.

[85] Brink T, Yesavage J, Owen L, et al. Screening tests for geriatric depression. Clin Gerontol. 1982;1:37-43.

[86] Ertan T, Eker E. Reliability, validity, and factor structure of the geriatric depression scale in Turkish elderly: are there different factor structures for different cultures? Int Psychogeriatr. 2000;12:163-172.

[87] Pfeffer R, Kurosaki T, Harrah C, Chance J, Filos S. Measurement of functional activities in older adults in the community. J Gerontol. 1982;37: 323-329.

[88] Selekler K, Cangöz B, Karakoç E. İşlevsel Faaliyetler Anketi'nin 50 yaş ve üzeri grupta Türk Kültürü İçin Uyarlama ve Norm Belirleme Çalışması. Türk Nöroloji Dergisi. 2004;10:102-107.

[89] Karakaş S. Bilnot Bataryası El Kitabı: Nöropsikolojik Testler İçin Araştırma ve Geliştirme Çalışmaları. Dizayn Ofset: Ankara, Turkey; 2004.

[90] Davis R, Ohman J. Wayfinding in ageing and Alzheimer's disease within a virtual senior residence: study protocol. $J$ Adv Nurs. 2016;72: 1677-1688.

[91] Davis R, Ohman J, Weisbeck C. Salient cues and wayfinding in Alzheimer's disease within a virtual senior residence. Environ Behav. 2017;49: 1038-1065.

[92] Kelly F, Innes A, Dincarslan O. Improving care home design for people with dementia. J Care Services Manag. 2011;5(3):147-155.

[93] Marquardt G. Wayfinding for people with dementia: a review of the role of architectural design. HERD: Health Environ Res Design J. 2011;4(2): 75-90.

[94] Chebat J, Morrin M. Colors and cultures: exploring the effects of mall Décor on consumer perceptions. J Bus Res. 2007;60(3):189-196.

[95] Fehrman K, Fehrman C. Color: The Secret Influence. 2nd ed. Upper Saddle River, NJ: Pretice Hall; 2004.

[96] Jalil N, Yunus R, Said N. Environmental colour impact upon human behaviour: a review. Procedia Soc Behav Sci. 2012;35:54-62.

[97] Allen M, Hogeland R. Spatial problem-solving strategies as functions of sx. Percept Mot Skills. 1978;47:348-350.
[98] Castelli L, Corazzini L, Geminiani G. Spatial navigation in large-scale virtual environments: gender differences in survey tasks. Comput Hum Behav. 2008;24:1643-1667.

[99] Coluccia E. Learning from maps: the role of visuo-spatial working memory. Appl Cogn Psychol. 2007;22:217-233.

[100] Lawton C. Gender differences in way-finding strategies: relationship to spatial ability and spatial anxiety. Gender Roles. 1994;30(11-12): 765-779.

[101] Linn M, Petersen A. Emergence and characterization of gender differences in spatial ability: a meta-analysis. Child Dev. 1985;56: 1479-1498.

[102] McGee M. Human spatial abilities: psychometric studies and environmental, genetic, hormonal, and neurological influences. Psychol Bull. 1979; 86:889-918.

[103] Moffat S, Hampson E, Hatzipantelis M. Navigation in a virtual maze: sex differences and correlation with psychometric measures of spatial ability in humans. Evol Human Behav. 1998;19(2):78-87.

[104] Sevinc Z, Bozkurt E. Wayfinding behaviors in a healthcare environment: a case study analysis of individual differences. Gazi University Journal of Science. Part B Art Humanities, Design Plan. 2015;3(3):37-45.

[105] Suzer O, Olgunturk N, Guvenc D. (2018). The effects of correlated colour temperature on wayfinding: a study in a virtual airport environment. Displays. 2018;51:9-19.

[106] Chebat J, Chebat C, Therrien K. Gender-related wayfinding time of mall shoppers. J Bus Res. 2008;61:1076-1082.

[107] Kozlowski L, Bryant K. Sense of direction, spatial orientation, and cognitive maps. J Exp Psychol Hum Percept Perform. 1977;3:590-598.

[108] Vandenberg S, Kuse A, Vogler G. Searching for correlates of spatial ability. Perceptual Motor Skills. 1985;60:343-350.

[109] Lawton C, Kallai J. Gender differences in wayfinding strategies and anxiety about wayfinding: a cross-cultural comparison. Sex Roles. 2002; 47(9-10):389-401.

[110] Silverman I, Choi J, Peters M. The hunter-gatherer theory of sex differences in spatial abilities: data from 40 countries. Arch Sex Behav. 2007; $36: 261-268$.

\section{AUTHORS' BIOGRAPHIES}

OZGE KUMOGLU SUZER is currently working as an interior designer at Lorne Rose Architect in Toronto, Canada. She received a BFA in Interior Architecture and Environmental Design Department of Bilkent University, Ankara, in 2011. After completing her BFA, she continued her studies focusing on the field of environmental psychology and lighting design, and a MFA in 2013. She is currently on the last steps of her PhD candidacy in Bilkent University. While continuing her MFA and $\mathrm{PhD}$ studies, she also worked as a research and teaching assistant in Bilkent University (2011-2015) and Atilim University (2015-2017). She is an active member of the Inter-Society Color Council (ISCC) and the intern member of the Association of Registered Interior Designers of Ontario (ARIDO).

NILGUN OLGUNTURK is an associate professor in the Department of Interior Architecture and Environmental Design, the Faculty of Art, Design and Architecture, Bilkent University, Ankara. She received her BArch, M.A., and Ph.D. degrees in architecture. Her professional experience has included appointments as instructor at the Middle East Technical University and research fellow at the London South Bank University. She has worked on research projects in the UK for 
NHS Estates (Department of Health) on color design in hospitals and EPSRC/DTLR LINK (Department of Transport, Local Government and the Regions) on color, visual impairment, and transport environments. She has 20 years of research experience on color perception, color preference and color use in architecture. Her current research and teaching include color and lighting, undergraduate and graduate studios. Dr. Olguntürk is among others, an active member in the International Commission of Illumination (CIE), the International Colour Association (AIC), the Inter-
Society Color Council (ISCC) and the UIA, Chamber of Architects of Turkey.

How to cite this article: Süzer ÖK, Olguntürk N. The aid of colour on visuospatial navigation of elderly people in a virtual polyclinic environment. Color Res Appl. 2018;43:872-884. https://doi.org/10.1002/col. 22272
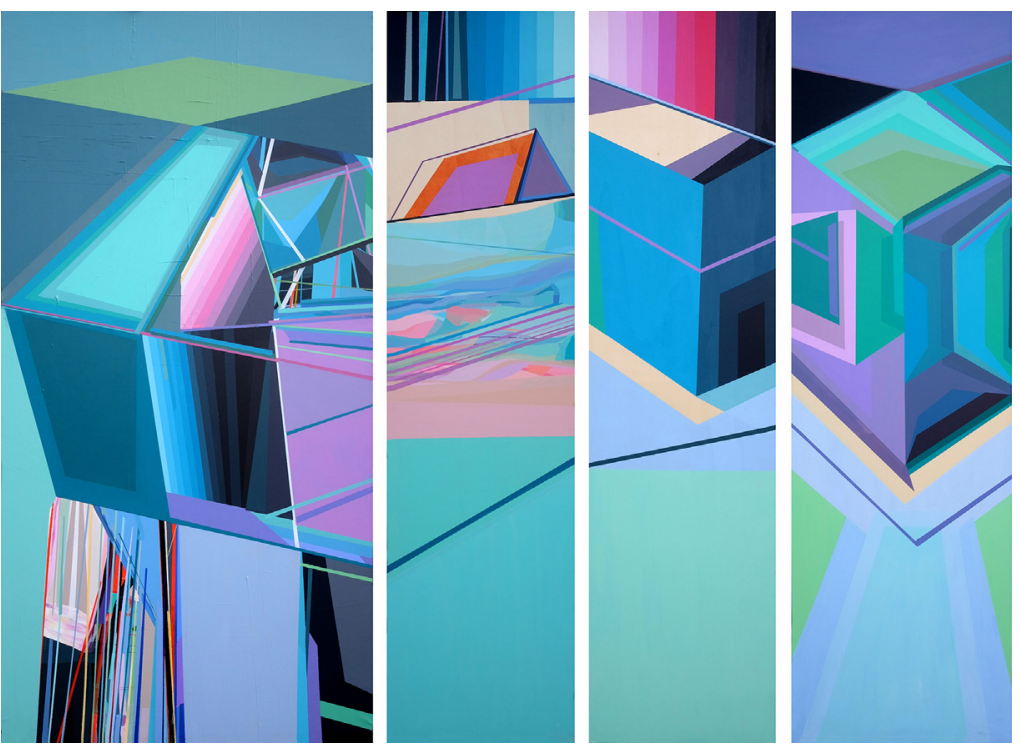

ANA PAIS OLIVEIRA

PORTUGAL

Title: Ar Livre \#2

Media: acrylic on canvas and maritime plywood 